following found.-Very extensive abscess of the frontal lobe of the right side of the brain. No signs of fracture of the orbital plate of the frontal bone or of the ethmoid bone. There were patches of meningitis on the dura mater of the right side. In short, the frontal lobe was broken down and bathed in a large quantity of pus.

\title{
Remarks
}

In conclusion, I may say these two cases point to the necessity of making free incisions into the orbit early where cellulitis is suspected and of not waiting for "pointing" and a feeling of fluctuation. Further, if the skull is opened, an attempt should be made to open as close to the site of injury as possible.

\section{A NOTE UPON THE PSEUDO-NEOPLASTIC FORM OF INTERSTITIAL KERATITIS}

\author{
BY \\ Sydney Stephenson, \\ LONDON.
}

WHILE reading the account given by Dr. F. A. Marchi in the November (1916) number of the Archivio di Ottalmologia of a couple of cases where trachomatous pannus appeared under the guise of a tumour (for abstract see THE BRITISH JOURNAL OF OPHTHALMOLOGY, September, 1917), I was reminded of an appearance seen, although rarely, in cases of interstitial keratitis. I have met with but few instances of this striking and curibus clinical picture, but have little doubt that others, besides myself, are familiar with it, although, so far as I know, it has not been specially described before.

The cases have all occurred in young subjects, who presented some of the ordinary stigmata of hereditary syphilis. Once only have I seen both corneae affected at the same time, although on several occasions, in my own experience, the condition has occurred first in one eye and then in the other. The clinical picture has been always the same, and may be briefly outlined as follows.-Occupying the upper or the lower part of the cornea, in the position of a so-called "Hutchinson's salmon patch," has been a somewhat prominent, fleshy-looking mass, at first sight resembling nothing so much as a neoplasm. The cornea adjoining the central part of the edge of the mass has shown interstitial deposits, or in more advanced cases, the entire cornea, not occupied by the growth, has exhibited the appearances of aggressive interstitial keratitis. In course of time the fleshy-looking mass has lost its prominence, so 
that the part upon which it was situated became flush with the rest of the cornea. It was followed by no local bulging. So far as I have observed, cases of the kind described run much the same course as the ordinary ones of interstitial keratitis. They are, perhaps, rather more tedious, but on that point I should not care to express a positive opinion.

When both corneae are affected, the diagnosis can offer few difficulties, for bilateral tumours must be among the greatest of rarities. When one eye alone is involved, the mass may be mistaken for a papilloma, a sarcoma, or other form of new growth. The difficult cases are those in which the pseudo-neoplasm forms the earliest sign of the disease. Points that would assist in reaching a correct diagnosis are the normal appearance of the neighbouring conjunctiva, the age of the patient, the history of the case, the concomitant evidences of syphilis, and the course of the affection.

An account is given below of two cases of the kind:-

1. Gertrude P., at. 9 years, a child with an obviously syphilitic physiognomy, was seen in October, 1914, on account of her right eye, which had been inflamed for about a month. The affected eye was reddened and very sensitive to light, and when the lids were separated, the child sneezed violently. The upper third of the cornea was occupied by a prominent red mass, and the remainder of that structure was pervaded by greyish spots of opacity, lying at different levels. These opacities were most marked near the edge of the red mass. The surface of the cornea looked dull, as if it had been breathed upon. Under treatment by mercury and chalk and the local use of atropin, the right cornea improved a good deal, but in December, 1914, the left cornea became involved and showed much the same clinical appearances as the right had done when I first saw the patient. After several months' treatment, the fleshy-looking masses disappeared, and the corneae resembled those of a receding case of interstitial keratitis. The child was seen towards the end of 1915 , when V. was $6 / 18$, there was no keratectasia, and the corneae had to a large extent recovered their transparency, although Hirschberg vessels were found on dilatation of the pupils and examination with a magnifying glass.

2. May F., at. 15 years, seen on July 24, 1907, with the history that the right eye had been inflamed for two weeks. From the hospital records I found that this girl had attended from December 20, 1905, to August 25, 1906, on account of an attack of interstitial keratitis affecting the left cornea. The evidence of hereditary syphilis included pegged teeth and rhagades about left side of mouth. Now, on examining the right eye, it was found that the lower third of the cornea was occupied by a salient "salmon patch" of the most typical kind. The cornea immediately. 
above the patch was more or less opaque, although the upper half, say, of the structure was clear. $V .=5 / 6$. Intra-ocular pressure was slightly lowered. The condition was treated by occlusion of the eye, mercury with chalk internally, and locally atropin and dionin. On August 14, 1907, R.V.=hand-reflex. T.n. There was scarcely any pain. The upper and inner part of the cornea was occupied by a salmon patch, and the ectatic salmon patch already described was still present. On September 4, 1907, the right cornea was wholly vascularized, save for an oval area just above the centre, through which the outline of the pupil could be recognized. Three weeks later, R.V.=hand-reflex. T.n. Vascularity of cornea reduced, but cornea so opaque that no trace of the pupil could be seen. On November 27, 1907, R.V.=fingers at one metre. There was a dense central corneal opacity of irregular outline. The remainder of the cornea was hazy and vascular, with $(a)$ a slight interstitial plaque of pinkish colour of the lower third, and $(b)$ a vascularized infiltration of the upper and inner part.

\section{ANNOTATIONS}

\section{Ophthalmia Neonatorum}

The necessity of making proper provision in large towns for the collective treatment of cases of ophthalmia neonatorum has been often emphasized. Steps have already been taken in that direction in Liverpool and in Glasgow. As regards London (where five beds only appear to be available for such cases) Mr. E. Treacher Collins in the course of evidence given not long ago before the Royal Commission on Venereal Diseases suggested that the whole matter should be handed over to the Metropolitan Asylums Board, the necessary powers being given for the purpose. The matter has now been carried a step further, largely owing to the recent Report of the Departmental Committee on the Welfare of the Blind. The Local Government Board has asked the Metropolitan Asylums Board to make provision for the hospital treatment of certain cases of the disease. It is suggested that it would suffice if at the outset two hospitals or two special departments of existing hospitals were provided, one for North and the other for South London. It is further suggested that a total accommodation for about fifty cases would be ample. In Liverpool, it may be recalled, provision for these cases is made by St. Paul's Eye Hospital, where special wards are provided, and in Glasgow by the city authorities. Under either scheme the object aimed at is to avoid separating mother from baby. We trust this point will not be overlooked in any provision that may be made in London. 